René Játiva, E. Garzón and J. Vidal, “Space-Time Diversity for NLOS Mitigation in TDOA-Based Positioning Systems", IEEE International Engineering Summit, II Cumbre Internacional de las Ingenierías, March 2016. DOI: 10.1109/IESummit.2016.7459771. 


\section{Space-Time Diversity for NLOS Mitigation in TDOA-Based Positioning Systems}

\author{
René Játiva E.*, Esteban Garzón \\ Colegio de Ciencias e Ingeniería \\ Universidad San Francisco de Quito (USFQ) \\ Quito, Ecuador \\ rjativa@usfq.edu.ec
}

\author{
Josep Vidal \\ Departamento de Teoría de la Señal y Comunicaciones \\ Universidad Politécnica de Cataluña \\ Barcelona, España \\ josep.vidal@upc.edu
}

\begin{abstract}
This paper studies the potential impact of using space-time information in the mitigation of the Non-Line-OfSight condition in mobile subscriber's positioning systems. First of all, this work discusses the positioning problem based on measures of Time Differences Of Arrival departing from a more exact characterization of the signal statistics and including some geometrical restrictions to achieve an improved accurate. Furthermore, a novel approach that integrates signal propagation characteristics to information provided by a suitable timing estimation model based on Cramer Rao Bound for a Rayleigh-fading channel, when antenna arrays are used at the receiver and when a set of channel vector estimates are available, has been introduced to study the positive benefits of space-time diversity. These approaches are evaluated within a realistic simulation scenario.
\end{abstract}

Keywords-NLOS mitigation, TDOA based positioning systems; WLLS; geometrical restrictions; wireless sensor network, Cramer Rao Bounds (CRB), Antenna Arrays.

\section{INTRODUCTION}

Positioning of a mobile subscriber is a complex task that adds value to services and applications and strongly impacts to our society. Subscriber positioning provides new possibilities of relation among users, between users and service providers, and also between providers and third parties. Therefore a close relationship and dynamism are associated to wireless communications that provide user ubiquity, positioning technologies that brings the location, and Location Based Services (LBS) that take advantage of these virtuosities. Being this the case, it is not strange that all these systems' elements are object of permanent research and revision [1]-[4].

Despite mobile subscriber positioning is mainly computed on mobile terminals nowadays, network based positioning is also important, for example in the use of minimization of drive test (MDT) [1] for network self-organization. This paper precisely focuses in network based positioning technologies and particularly in the mitigation of Non Line Of Sight (NLOS) propagating conditions, which strongly degrades the accuracy of position estimation, based on Time Differences Of Arrival (TDOA) measures in a realistic simulation environment.

Furthermore, and since current and future communication systems are equipped with antenna arrays, providing Multiple Input - Multiple Output (MIMO) capabilities, this work integrates a Cramer Rao Bound (CRB) model for timing, to a link level propagation simulator to gain insight to the possibilities of using a collection of space-time data as a mean to improve the timing estimation, and therefore achieve a better NLOS mitigation in subscriber positioning.

\section{The Positioning Problem}

In a passive location system, the measurements vector $\mathbf{m}$, may be viewed as a function of the position vector $\mathbf{x}$, plus additive noise $\mathbf{n}$, as in (1):

$$
\mathbf{m}=f(\mathbf{x})+\mathbf{n}
$$

The actual nature of $f(x)$ depends of the type of the measurements' set used for computing the positioning, and in the case of range-based methods such as TOA, TDOA and RSSI, it is a nonlinear function related to the range among the subscriber position and those BS's participating in the positioning. The expression for TDOA is exhibited in (2):

$$
f_{\mathrm{TDOA}_{i}}(\mathbf{x})=\left\|\mathbf{x}-\mathbf{r}_{i}\right\|-\left\|\mathbf{x}-\mathbf{r}_{1}\right\| ; \quad \forall i=2,3, \ldots L
$$

Where $\mathrm{L}$ refers to the number of BSs, $\mathbf{x}=(\mathrm{x}, \mathrm{y})$ to the true coordinates of the subscriber position, $\mathbf{r}_{\mathrm{i}}=\left(\mathrm{x}_{\mathrm{i}}, \mathrm{y}_{\mathrm{i}}\right)$ denotes to the position of $\mathrm{BS}_{\mathrm{i}}$ involved in the measure, $\mathbf{r}_{1}=\left(\mathrm{x}_{1}, \mathrm{y}_{1}\right)$ notes to the central site $\mathrm{BS}_{1}$ used as a common reference for each TDOA measure, and ||.|| to the Euclidean norm.

General nonlinear solutions are Maximum Likelihood (ML), Nonlinear Least Squares (NLS) or the Weighted Nonlinear Squares (WNLS) approach [5]. The WNLS solution requires minimization of a cost function, and performing $\mathrm{ML}$ estimation requires noise statistics. When the measurement noise $\mathbf{n}$ in (1) is zero-mean and Gaussian distributed with covariance matrix $\mathbf{C}_{\mathbf{n}}$, ML scheme reduces to the WNLS solution. The ML approach requires a high complexity when grid search is performed, and therefore global solution may not be guaranteed, but in general its accuracy is the highest, especially when $\mathbf{C}_{\mathbf{n}}$ is also a function of subscriber position [6]. However, having a perfect error statistical characterization is difficult and others approaches are preferred. On the other hand, NLS does not require noise statistics but also involves the same issues as ML.

The vector function $f(\mathbf{x})$ in (1) may be linearized through a Taylor series expansion around an arbitrary point $\mathbf{x}_{\mathbf{0}}$ located 
near the subscriber position. This procedure requires the Jacobian matrix for $\mathbf{f}(\mathbf{x})$ evaluated in $\mathbf{x}_{\mathbf{0}}$, for the set of measurements and the two coordinates in this case [6][7].

Assuming a zero mean Gaussian distribution for the noise vector n, Torrieri solution [7] uses the iterative algorithm known as Gauss-Newton method for reaching the cost function minimum, but others methods such as Newton-Raphson or Steepest Descent may be used instead [6]. It is also possible to convert the nonlinear formulation in (1) into a set of linear equations as in (3) under the assumption that measurement errors are small enough.

$$
\mathbf{A x}=\mathbf{b}
$$

Expressions (4) and (5) exhibit the corresponding structures for the elements in these matrices in case of TDOA based positioning systems when the controlling BS is assumed to be at the origin, and where $c$ refers to the light speed.

$$
\begin{gathered}
\qquad\left[\mathbf{a}_{i}\right]=\left[\mathbf{r}_{i+1}\right] ; \forall i=1,2, \ldots, L-1 \\
{\left[b_{i}\right]=\left[\frac{1}{2}\left(\left\|\mathbf{r}_{i+1}\right\|^{2}-m_{i+1}^{2}\right)-r . m_{i+1}\right]} \\
\text { with } \quad r=\|\mathbf{x}\| \text { and } m_{i}=c \tau_{i, 1} ; \quad \forall i=2,3, \ldots, L
\end{gathered}
$$

Linear procedures include to Linear Least Squares (LLS), Weighted LLS (WLLS) and the subspace estimators [5][6]. Since LLS dismisses noise statistics, the WLLS approach emerges by including a weighting matrix $\mathbf{W}$, within the cost function as it is shown in (6):

$$
J_{W L L S}=E\left\{(\mathbf{A x}-\mathbf{b})^{T} \mathbf{W}(\mathbf{A x}-\mathbf{b})\right\}
$$

This assessment matrix is precisely the inverse of the covariance noise matrix, and after cost function minimization in (6), the WLLS estimator is achieved as in (7):

$$
\hat{\mathbf{x}}=\left(\mathbf{A}^{T} \mathbf{W} \mathbf{A}\right)^{-1} \mathbf{A}^{T} \mathbf{W b}
$$

The particular weighting matrix $\mathbf{W}$ depends of the type of measurements, and it is usually dependent of the distances between subscriber and BSs due to transformations performed during formulation of the linear system. In (5), the term $b_{i}$ includes squared measures and therefore introduce a cross term of the range measure error with its own range. Furthermore, expression in (8) exhibits this matrix for the case of TDOA based positioning [5]:

$$
\begin{aligned}
& \mathbf{W}=\left[E\left\{\mathbf{e e}^{T}\right\}\right]^{-1} \\
& =\left[\operatorname{4diag}\left(d_{2}, d_{3}, \ldots, d_{L}\right) \mathbf{C}_{\mathbf{n}, T D O A} \operatorname{diag}\left(d_{2}, d_{3}, \ldots, d_{L}\right)\right]^{-1} \\
& \text { with } \quad d_{i}=\left\|\mathbf{x}-\overrightarrow{\mathbf{r}}_{i}\right\| ; \quad \forall i=2,3, \ldots, L
\end{aligned}
$$

But it says nothing about the nature of the noise covariance matrix $\mathbf{C}_{\mathbf{n}, \text { TDOA }}$. In the case of TOA measures, the set of required distances $d_{i}$ in (8) may be replaced by the set of measurements $m_{i}$ to solve the problem, but in case of using TDOA or AOA, a LLS procedure is usually firstly performed to estimate subscriber position to properly figure out the distances required within $\mathbf{W}$, and a second step is also required to achieve the refined WLLS position estimation. Our approach prefers however the use of WLLS even for initialization, as it will be explained later. Furthermore, an iterative process may be performed in order to minimize the function cost in (6) and achieve the maximum accuracy, and consequently the Best Linear Unbiased Estimator (BLUE) algorithm, but in general a two-step LS algorithm is adequate. Alternative formulations for the positioning problem are possible, but improvement in terms of accuracy is not important [8].

Whichever would be the positioning technique employed, it should be kept in mind that every set of measurements performed by a sensor reduces the positioning to a region shaped in a way related to the nature of the measurements, a feasible region. A TOA based positioning corresponds to a circular-circular system, whilst a hyperbolic-hyperbolic system is characteristic of a TDOA based positioning. Furthermore, it must be noted that hybrid techniques exhibit a better behavior than homogeneous ones since it is a well-known principle that errors achieved from a particular positioning technique may be overcome with the application of another one. Positioning accuracy may also take advantage of spatial diversity and mobile system's dynamic [9]. In fact, Kalman Filter and its variants have their efficacy by using the mobility dynamics.

\section{Signal Model And The NLOS Issue In The Positioning PROBLEM}

Due to the presence of obstacles in the scenario, received signal is scattered in space and time, and the LOS component may be strongly degraded or even completely shadowed. However, receiver generally uses the most powerful arriving components and therefore, in case of shadowing, LOS component is eventually discarded, and measures are achieved under a NLOS condition. This NLOS multipath signal travels a longer distance than the LOS component to reach the receiver and consequently the measures are biased as it is shown in (9):

$$
\begin{aligned}
& \mathbf{m}=f(\mathbf{x})+\mathbf{n}+\mathbf{q} \\
& \text { with } \quad \mathbf{q}=\left[q_{1}, q_{2}, \ldots, q_{L}\right]^{T}
\end{aligned}
$$

Where, $\mathbf{q}$ is precisely the vector which contains biases due to NLOS. These biases are positive random variables for TOA measures, but it is not necessarily the case for TDOA. When $\mathrm{q}_{\mathrm{i}}=0$, it refers to a LOS condition, and when $\left|\mathrm{q}_{\mathrm{i}}\right|>>\left|\mathrm{n}_{\mathrm{i}}\right|$, it refers to a strong NLOS condition, being the latter the case commented along this paper. Bias nature is associated to propagation conditions, and in case of timing based systems, it may be related directly to the Excess Delay through the Power Delay Profile (PDP). Furthermore, the Greenstein model [3] has been considered to perform this characterization, since it adjusts to several measurement-based models and incorporates their information into a small number of parameters to characterize the path-gain/delay spread propagation channel, and even this model has been incorporated to COST-231 and eventually to the more complex COST-259 Directional Channel Model [4].

NLOS environments are modeled using an exponential distribution for the excess delay as it is shown in (10): 


$$
f_{\tau}(\tau)=\frac{1}{\tau_{r m s}} \exp \left(-\frac{\tau}{\tau_{r m s}}\right) u(\tau)
$$

And the Greenstein model characterizes the required RMS Delay Spread $\tau_{\text {rms }}$ as a random variable and also as a function of the distance between emitter and receiver, as in (11):

$$
\boldsymbol{\tau}_{r m s}=T_{1} d^{\varepsilon} \boldsymbol{\xi}
$$

Where, $\xi$ is a lognormal random variable. Hence, $\boldsymbol{\Xi}=10 \log (\xi)$, is a zero mean Gaussian variable over the terrain, with a standard deviation $\sigma_{\xi}$ that lies between 2 and $6(\mathrm{~dB})$. Furthermore, $\mathrm{T}_{1}$ corresponds to the median value of $\boldsymbol{\tau}_{\text {rms }}$ at $\mathrm{d}=1$ $(\mathrm{km})$, and $\varepsilon$ is an exponent that lies between 0.5-1.0. It has been set to 0.5 for the simulations exhibited in this document.

The Greenstein model also includes the path gain g. This gain is related with the shadowing, changes stochastically and it is also distance dependent due to propagation conditions. This gain is computed as in (12):

$$
\mathbf{g}=\frac{G_{1}}{d^{\beta}} \mathbf{x}
$$

Where, $d$ is the distance in kilometers, $G_{1}$ is the median value of $\mathbf{g}$ at $\mathrm{d}=1(\mathrm{~km}), \beta$ is the loss path propagation factor which lies between 3 and 4 in urban areas, and $\mathbf{x}$ is a lognormal random variable. Therefore, $\mathbf{X}=10 \log (\mathbf{x})$ is a zero mean Gaussian with a standard deviation $\sigma_{\mathrm{x}}$ between 6 and $12(\mathrm{~dB})$. Finally, the correlation factor between $\mathbf{X}$ and $\boldsymbol{\Xi}$ has been set as $\rho=-0.7$ [3]. Therefore $\mathrm{E}\{\mathbf{X}\}=\rho . \sigma_{\mathrm{x}} . \sigma_{\xi}$. These path gains have also been incorporated in our simulations, where the pathgain/delay spread information has been related to the problem geometry to compute SNR at the receivers for each subscriber position at the cell. These realizations for SNR and delay spread have been provided to the CRB model for the timing estimation, commented in the next section, as a mean to study the potential impact of using antenna arrays in subscriber positioning.

The mean and the standard deviation for the RMS Delay Spread are presented as a function of distance in (13) and (14) respectively:

$$
\begin{gathered}
E\left\{\boldsymbol{\tau}_{r m s}\right\}=T_{1} d^{\varepsilon} e^{m_{z}+\sigma_{2}^{2} / 2} \\
\sigma_{\tau_{r m s}}=\sqrt{\operatorname{var}\left\{\tau_{r m s}\right\}}=E\left\{\boldsymbol{\tau}_{r m s}\right\} \sqrt{e^{\sigma_{z}^{2}}-1}=k T_{1} d^{\varepsilon}
\end{gathered}
$$

Being, $\mathrm{m}_{\mathrm{z}}$ and $\sigma_{\mathrm{z}}$ the mean and standard deviation of the scaled random variable $\mathbf{Z}=\boldsymbol{\Xi} \cdot \ln (10) / 10$. These expressions were derived in [10] and they will help us to construct the noise correlation matrix $\mathbf{C}_{\mathrm{n}}$ in (8), and the weighting matrix required in (7) to achieve the positioning.

Reminding that both the independent vector in (5) and also the weighting matrix in (8) require the knowledge of the subscriber's position, this information is initially regarded. Therefore, $r$ in (5) is set to zero, and the weighting matrix $\mathbf{W}$ in (8) discards its dependence with the distance, and becomes $\mathbf{W}^{\mathbf{0}}$, as it is shown in (15):

$$
\mathbf{W}^{o}=\left[\mathbf{C}_{\mathbf{n}}^{o}\right]^{-1}
$$

Setting $r$ to zero at initialization is implicitly telling that subscriber is near the central site, whilst the use of the weighting matrix in (15) informs that there is not a priori preferred position over a radial basis around this site. Furthermore, since signal fades independently for each link between the subscriber and the base stations, and in the absence of a priori information, timing offsets may be considered as independent and identically distributed random variables; and since TDOA measures share a common reference to the central site, these measures keep some cross correlation. Therefore, the noise covariance matrix takes the form in (16) where offdiagonal elements are the half of the terms in the diagonal.

$$
\left[\mathbf{C}_{\mathbf{n}}^{o}\right]_{i i}=2 ; \quad\left[\mathbf{C}_{\mathbf{n}}^{o}\right]_{i j}=1 ; \quad \forall i \neq j
$$

The resulting position $\mathbf{x}^{0}$ from the use of (15) and (16), and $r=0$ is used to estimate the set of distances $d_{i}$ required in (8), and for the computation of the error covariance matrix $\mathbf{C}_{n}$ that is also distance dependent as it is easily verifiable from (13) and (14). Therefore $\mathbf{C}_{\mathrm{n}}$ takes the form in (17) by discriminating the error contribution associated to each independent link.

$$
\left[\mathbf{C}_{\mathbf{n}}\right]_{i i}=T_{1}^{2}\left(d_{i}^{2 \varepsilon}+d_{1}^{2 \varepsilon}\right) ; \quad\left[\mathbf{C}_{\mathbf{n}}\right]_{i j}=T_{1}^{2} d_{1}^{2 \varepsilon} ; \quad \forall i \neq j
$$

Certainly, a more exact characterization of $\mathbf{C}_{\mathrm{n}}$ is possible, but such solution does not just require the knowledge of $\sigma_{z}$, but also has a more complex form that includes cross products of distances, and since estimates are being used instead of the true lengths, the additional burden is not justified by the final resultant marginal improvement.

Due to $\mathbf{x}^{0}$ is a poor estimate, $r$ is set to 0 again, and (17) is now used to construct $\mathbf{W}$ as in (8), and hence a better solution $\mathbf{x}^{1}$ is achieved. This estimation is then used to compute $r$, as $\mathbf{r}=\left\|\mathbf{x}^{1}\right\|$. Finally, the displacement vector is computed from (5) and (7), and it is added to the solution to get the positioning.

When studying the results, some degradation was observed near the cell boundaries, so a geometrical restriction was introduced to ensure that positioning is achieved within the control site. The performance of this proposal is evaluated in section VI.

\section{Model For The First Arrival Based On The Cramer RAO BOUND}

The Greenstein model mentioned in the section above characterizes the signal time dispersion by an exponential distribution defined by a random delay spread. At the receiver, the most powerful arrival is chosen to attain reliable communications, and this path does not correspond in general to the first arrival. Therefore, this timing is usually biased and follows an exponential distribution. However, it is possible to look for the first arrival by performing a hypothesis test and by using high resolution spectral techniques for example, and consequently improve timing estimation. This is conceivable since in a very short time, multiple channel vector estimates may be collected whilst subscriber positioning practically remains invariant. 
The quality of these first-arrival estimates improves with diversity, and it can be studied with the Cramer Rao Bound (CRB) [9], the Barakin Bound (BB) [11] or the Ziv-Zakai Bound (ZZB). However CRB is adequate to model Gaussian processes in case of having a large record of estimates [11]. $\mathrm{CRB}$ is an inferior bound of the variance in the estimation of a parameter, in this case the estimation of the first arrival timing. Therefore, it is an optimistic model that shows the quality of the best possible estimation; however CRB does not tell the way to achieve such optimal estimator.

Our CRB model has been better explained in [9] but their main characteristics are commented below. This model has been preferred over other similar in the literature since it takes account for both the spatial and temporal diversity of the channel estimates. It assumes the use of Direct Sequence Spread Spectrum (DS-SS) for the Data Aided timing estimation. It could be the case of W-CDMA for example, where a reference pseudo-random sequence known at the receiver is used for dispreading, and when the receiver is equipped with an antenna linear array. Therefore, the resultant signal $y_{s}{ }^{(j)}(k)$ is a channel estimate described in (18).

$$
y_{s}^{(j)}(k)=\sum_{i=1}^{N_{\text {paths }}} b_{i j}(k) \gamma_{i}(k) e^{j 2 \pi f_{i} k} g\left(\tau_{s}-\tau_{i}\right)+w_{s}^{(j)}(k)
$$

Where, "s" subscript refers to the lag within the observed window, " $j$ " superscript denotes the sensor within the array, and " $k$ " identifies the time slot when the estimate was performed. Furthermore, " $i$ " identifies the multipath component, $b_{i j}$ refers to the spatial signature, $\gamma_{i}$ to the power path gain, $f_{i}$ to the Doppler component, $g($ ) denotes the pulse shape, and $\tau_{i}$ the timing for the path; whilst $w_{s}{ }^{(j)}(k)$ denotes the estimation noise.

Arranging lag estimates in a vector, (18) turns into (19):

$$
\mathbf{y}^{(j)}(k)=\mathbf{G}_{s} \mathbf{b}^{(j)}(k)+\mathbf{w}^{(j)}(k)
$$

Where, $\mathbf{G}_{\mathrm{s}}$ is the pulse shape matrix described in [9], $\mathbf{b}^{(\mathrm{j})}$ is the spatial signature vector and $\mathbf{w}^{(\mathrm{j})}$ is the estimation noise vector. Furthermore, when a collection of these channel estimates for each sensor are re-arranged in a new vector $\mathbf{y}$, as it is shown in (20), this new vector may be modeled as circular Gaussian with a mean associated to the first arrival $\boldsymbol{\mu}_{\mathrm{y}}$, and a correlation matrix $\mathbf{R}_{\mathrm{y}}$.

$$
\begin{aligned}
& \mathbf{w}=\left[\begin{array}{lllll}
\mathbf{w}^{(1)}(1)^{T} & \ldots & \mathbf{w}^{(1)}(K)^{T} & \ldots & \mathbf{w}^{\left(N_{s}\right)}(K)^{T}
\end{array}\right]^{T} \\
& \mathbf{y}=\left[\begin{array}{lllll}
\mathbf{y}^{(1)}(1)^{T} & \ldots & \mathbf{y}^{(1)}(K)^{T} & \ldots & \mathbf{y}^{\left(N_{s}\right)}(K)^{T}
\end{array}\right]^{T}
\end{aligned}
$$

Analogously, the re-arranged error vector $\mathbf{w}$ will also be circular Gaussian, but with zero mean and with independent and identically distributed elements of variance $\sigma^{2}{ }_{w}$, as it is stated in (21).

$$
\mathbf{w} \sim C N\left(\mathbf{0}, \sigma_{w}^{2} \mathbf{I}\right), \quad \mathbf{y} \sim C N\left(\boldsymbol{\mu}_{y}, \mathbf{R}_{y}\right)
$$

The correlation matrix $\mathbf{R}_{\mathrm{y}}$ in (22), after some cumbersome work [9] can be expressed as in (23), due to space and time scattering processes may be decoupled:

$$
\begin{gathered}
\mathbf{R}_{y}=E\left\{\mathbf{y} \mathbf{y}^{H}\right\} \\
\mathbf{R}_{\mathbf{y}}=\mathbf{R}_{\phi}(\boldsymbol{\rho}) \otimes \mathbf{T}(\alpha) \otimes P_{s} \mathbf{G}_{s}(\beta) \boldsymbol{\Lambda}_{\boldsymbol{\tau}}\left(\lambda_{n}\right) \mathbf{G}_{s}{ }^{H}(\beta)+\sigma_{w}^{2} \mathbf{I}
\end{gathered}
$$

Where, $\mathbf{R}_{\phi}$ is the spatial correlation matrix among sensors, $\mathbf{T}$ is the temporal correlation matrix among channel estimates achieved at different time slots, and $\boldsymbol{\Lambda}_{\tau}$ is a diagonal matrix that models the power of arrivals with exponential decaying and parameter $\lambda_{n}$, being $P_{s}$ the power of the first arrival for a dispersive Rayleigh propagation model, as it is shown in (24).

$$
\left\{\boldsymbol{\Lambda}_{\tau}\right\}_{i, i}=\exp \left[-\left(i-k_{0}\right) \lambda_{n}\right] u\left(i-k_{0}\right)
$$

Since $\mathbf{R}_{\phi}$ is Toeplitz, it is characterized just by the first row, the vector $\boldsymbol{\rho}$. Temporal correlation depends of signal Doppler, and it has been modeled as a first order Autoregressive (AR) process where $\alpha$ is the temporal correlation between two consecutive slots. The particular form of the pulse shape matrix depends of the pulse shape $g(t)$. In our case, it is a raised cosine with roll off factor $\beta$. Furthermore, $\lambda_{n}$ is closely related to the coherence bandwidth and therefore to the signal delay spread. For the computation of the spatial correlation matrix $\mathbf{R}_{\phi}$, a spatially scattered signal around the Line Of Sight (LOS) bearing with a Gaussian distribution was considered.

With this model at hand, a vector of parameters $\boldsymbol{\Psi}$ is defined in (25):

$$
\boldsymbol{\Psi}=\left[k_{0}, \lambda_{n}, \beta, P_{s}, \sigma_{w}^{2}, \alpha, \boldsymbol{\rho}^{T}\right]^{T}
$$

And, since $\mathbf{y}$ in (20) is a zero mean circular Gaussian vector, the Fisher Information Matrix (FIM) $\mathbf{F}_{\boldsymbol{\Psi}}$ for these parameters is formulated as in (26).

$$
\left[\mathbf{F}_{\mathbf{\Psi}}\right]_{p q}=\operatorname{tr}\left(\mathbf{R}_{\mathbf{y}}^{-1} \frac{\partial \mathbf{R}_{\mathbf{y}}}{\partial \mathbf{\Psi}_{p}} \mathbf{R}_{\mathbf{y}}^{-1} \frac{\partial \mathbf{R}_{\mathbf{y}}}{\partial \boldsymbol{\Psi}_{q}}\right)
$$

Therefore, the Cramer Rao Bound for the timing estimation is extracted from the inverse of the FIM as is (27) [9].

$$
C R B\left(k_{0}\right)=\left[\mathbf{F}_{\boldsymbol{\Psi}}^{-1}\right]_{11} \leq \operatorname{var}\left\{\hat{k}_{0}\right\}
$$

\section{INCORPORATING FIRST ARRIVAL TIMING CRB}

Since the CRB for timing is sensitive to the SNR at the receiver and hence to the signal delay spread, the Greenstein model provides these parameters to the CRB model for each point within the simulation platform, to compute the lowest possible value for the timing variance in terms of representative parameters of the simulated environment, such as the Root Mean Square (RMS) angular spread, the channel temporal correlation, among others; and also of the network equipment configurations, such as the number of antennas and the number of available channel vector estimates used to improve timing estimation. However, it is well known that the CRB often fails at low SNRs due to the modeling usually doesn't include some a priori information [12]. Therefore, to avoid artificially creating a too optimistic scenario, especially for weak measures related to distant BSs, assuming erroneously that first arrival 
identification and its timing estimation is always possible, the square root of the CRB is compared with a threshold set to $50 \%$ of the chip time before deciding if the simulation accepts the new variance value. So when the CRB predicts a better behavior compared to the threshold, our model assumes a successful case where the predicted variance corresponds just to a Gaussian measurement error. On the contrary, when the quality of the estimate is not sufficient, the measure is possibly biased, and therefore, it follows an exponential distribution where error is computed as it was explained in section III. In addition, and to reduce excessive computational burden during simulations, reduced expressions for CRB in terms of SNR and DS were derived using a two-exponentials interpolation. These expressions introduce errors within 5\% respect the originals.

\section{Algorithms’ Performance Evaluation}

This section includes some simulations. The simulation platform is built upon a seven hexagonal cell cluster. The control site is located at the coordinate system origin, and a rectangular grid has been constructed within the control cell to evaluate subscriber positioning algorithms' behavior for each point within the cell. 1000 realizations for each position at the grid have been used to get the results. A realistic scenario where NLOS is present in the seven BS's, has been considered. The required parameters for the Greenstein model take the following values suitable for the urban case [3]: $\mathrm{T}_{1}=0.4$ (us), $\varepsilon=0.5, \quad \beta=3.7, \quad \sigma_{x}=8.0(\mathrm{~dB}), \sigma_{\xi}=4.0(\mathrm{~dB})$, and $\rho=-0.75$. Particularly, $\mathrm{T}_{1}$ has been set in agreement to the GTU COST 259 model [4] and it may be considered a moderate dispersive environment.

Fig. 1 exhibits the average positioning errors for subscribers within a cell of radius $\mathrm{R}=1000(\mathrm{~m})$ when WLLS algorithm is performed over UL-TDOA measures, and geometrical restrictions are applied. Average errors are lower than 250 (m) in most of the cases. This figure illustrates that the positioning accuracy improves near the central site and degrades near the cell boundaries in spite of the application of a geometrical restriction as a mean to reduce errors at the boundaries. In fact, when the algorithm notes that " $r$ " required in (4) takes a value higher than the cell radius, it sets $r=\mathrm{R}$, and compels the solution to be within the central site. Fig. 2 shows however as this refinement improves the positioning in at least 40 (m) for $70 \%$ of the cases and much more for the remainder, when measures are taken at the uplink (UL). For measures taken at the downlink (DL), improvement associated to geometrical restrictions is something lower, around 30 (m) for $70 \%$ of the cases. Fig. 2 also shows that positioning is systematically better for measures taken at the uplink (UL) due to in this case the whole set of TDOA measures uses the same timing reference for the central site. It does not occur at the DL, since Idle Period in Down Link (IPDL) is used in this case to perform the measure at the subscriber. Average positioning error reduces from 241 (m) for DL, to 211 (m) for UL for the $70 \%$ of the cases for our refined algorithm. However, it is important to keep in mind that SNR at DL is better than the corresponding at the UL due to power limitations at the mobile, previously to judge about these methods.

Fig. 3 shows the subscriber's average positioning error resulting from TDOA measures when the timing reference to the central site differs at each measure, when 16 sensors are available at the uniform linear array (ULA) in the receiver, and 50 channel vector estimates are used to improve the timing estimation. It could be the case of positioning based on DLTDOA. However, it is not probable to have so large array at the downlink. Nevertheless, this result is useful to show how positioning degrades near the central site, whilst remains uniformly well at the boundaries. This behavior is related with the fact that SNR keeps constant at the subscriber for the signal coming from the control site due the power control, whilst SNR degrades for signals coming from the surrounding BSs, and it is the so called near-far effect.

Fig. 4 shows the benefit of using space and temporal diversity. Note for example that positioning error reduces from $241(\mathrm{~m})$ at the original scenario to $153(\mathrm{~m})$ in the new one for the $70 \%$ of the cases, and from 287 (m) to 205 (m) for $95 \%$ of the cases.

POSITIONING ERROR - WLLS TDOA + G.R.

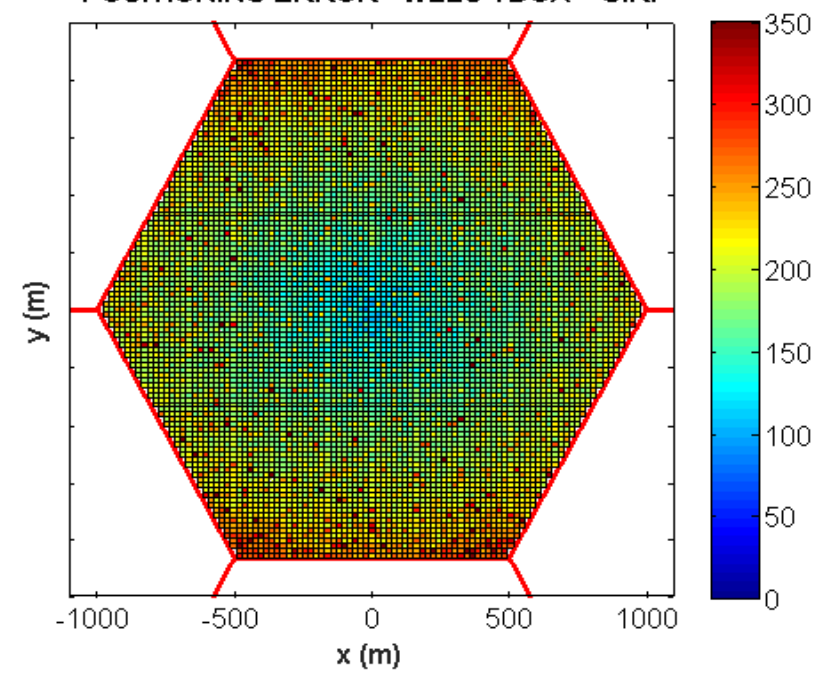

Fig. 1. Subscriber Average Positioning Error for a dispersive NLOS environment - UL-TDOA WLLS algorithm with 7 BSs and geometrical restrictions.

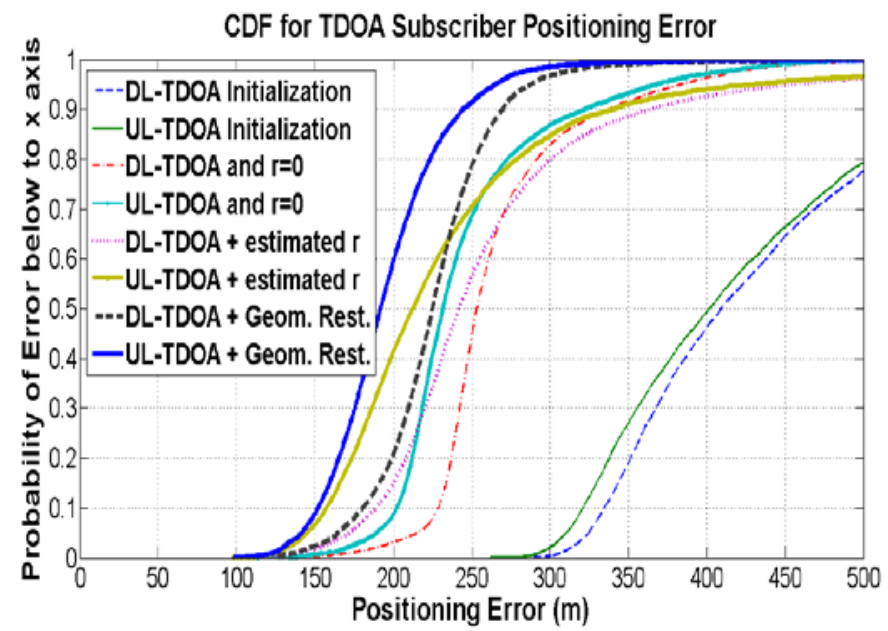

Fig. 2. Cumulative Distribution Function (CDF) for the Subscriber Positioning Error for a dispersive NLOS environment when WLLS algorithm is applied on TDOA measures taken at the uplink (UL- solid) or at the downlink (DLdashed) at different stages of the algorithm. 
Furthermore, when $\mathrm{K}=50$ channel vector estimates, and four sensors are used, average positioning improves in 20 (m) respect to the initial case for $70 \%$ of the cases, and improves another $30(\mathrm{~m})$ when the number of sensors doubles. When the number of sensors reaches sixteen a new improvement of 38 $(\mathrm{m})$ is achieved. Therefore an average positioning error below $116(\mathrm{~m})$ is achieve with 32 sensors for $70 \%$ of the cases, and below $181(\mathrm{~m})$ for the $95 \%$ of the cases. For positioning based on UL-TDOA, these errors reduce even more for the same configuration: to $76(\mathrm{~m})$ and $103(\mathrm{~m})$ respectively. When a larger number of channel vector estimates are available, great improvements can also be achieved. For example, with four sensors and $K=250$, positioning error reduces to $114(\mathrm{~m})$ for $95 \%$ of the cases for UL-TDOA. When just two sensors at the subscriber, for a higher SNR of $15(\mathrm{~dB})$, and $\mathrm{K}=250$, the positioning error is below to $126(\mathrm{~m})$ for $70 \%$ of the cases.

\section{POSITIONING ERROR - WLLS DL-TDOA, K=50, SNR=10(dB)}

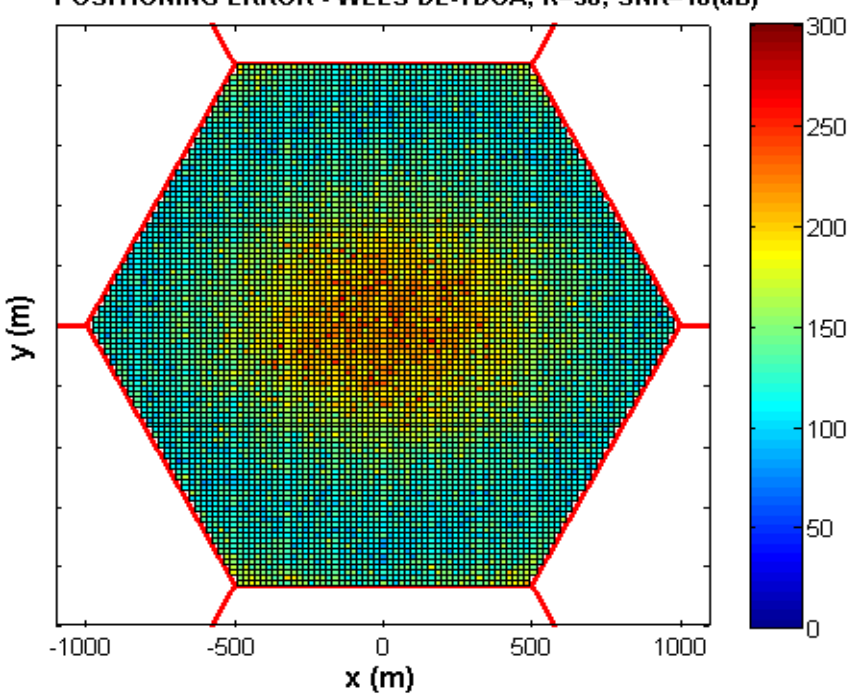

Fig. 3. Subscriber Average Positioning Error for a dispersive NLOS environment. The WLLS algorithm uses TDOA measures from 7 BSs, and adds geometrical restrictions for refinement. Timing for the central site differs for each measure. Receivers are equipped with 16 sensors - Uniform Linear Array (ULA), and 50 channel vector estimates are used to achieve the timing.

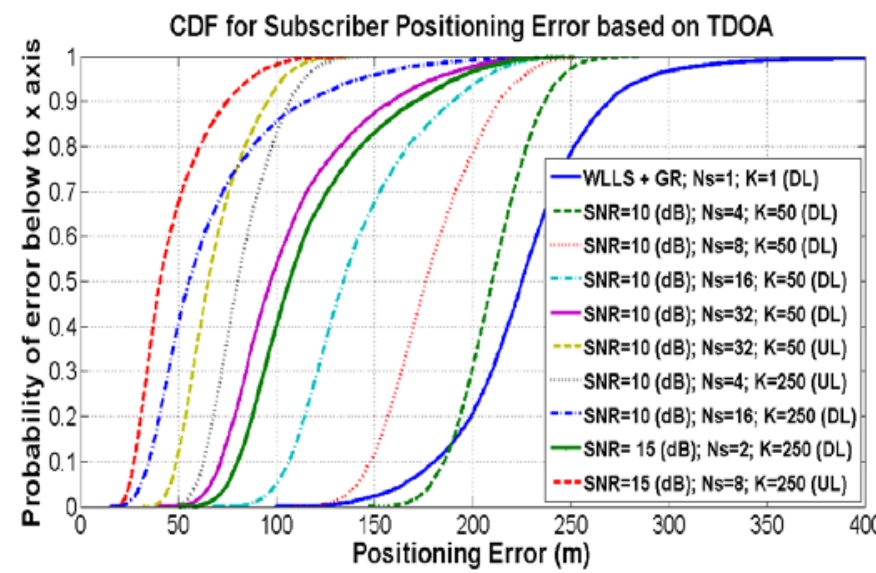

Fig. 4. Distribution Function (CDF) for the Subscriber Positioning Error in a dispersive NLOS environment when WLLS algorithm is applied on TDOA measures. Various scenarios are compared in terms of SNR, the number of sensors in the ULA at the receiver, and the way the measures are performed.

\section{SUMMARY}

NLOS condition strongly degrades the performance of subscriber positioning in wireless communication systems, and even robust algorithms, originally developed for LOS signal, fail. However, a better use of signal statistics to properly weigh the measures and imposing certain geometrical restrictions help to mitigate this problematic condition and therefore to improve positioning. Furthermore, a novel approach that integrates the simple but reliable link level Greenstein propagation model to a Rayleigh-fading model based on the CRB, that takes account of spatial and temporal diversity, has been provided to study the possibility of improving timing estimation to achieve a much better positioning. Our results show that important improvement is always possible for architectures that take advantage of temporal and spatial diversity, especially for positioning based on UL-TDOA.

\section{REFERENCES}

[1] Johan Johansson, Wuri A. Hapsari, Sean Kelley and Gyula Bodog, "Minimization of Drive Tests in 3GPP Release 11", IEEE Commun. Mag., vol. 50, no. 11, pp-36-43, November 2012.

[2] "Technical Specification Group Radio Access Network; Stage 2 functional specification on User Equipment (UE) positioning in UTRAN (Release 6) - 3GPP TS 25.305 V6.1.0 (2004-06)”, 3RD Generation Partnership Project, 2004.

[3] Larry J. Greenstein, Vinko Erceg, Yu Shuan Yeh, and Martin V. Clark, "A New Path-Gain/Delay-Spread Propagation Model for Digital Cellular Channels”, IEEE Trans. Veh. Technol., Vol. 46, No.2, pp. 477-485, May, 1998.

[4] Henrik Asplund, Andrés Alayón Glazunov, Andreas F. Molisch, Klaus I Pedersen and Martin Steinbauer, "The COST 259 Directional Channel Model - Part II: Macrocells”, IEEE Trans. Wireless Commun., Vol. 5, 12, 2006.

[5] Seyed A. Zekavat and R. Michael Buehrer, "Handbook of Position Location - Theory, Practice and Advances”, IEEE Press - John Wiley and Sons, 2012.

[6] Fredrik Gustafsson and Fredrik Gunnarsson, "Mobile Positioning using Wireless Networks - Possibilities and fundamental limitations based on available wireless network measurements”, IEEE Signal Process. Mag., Vol. 22, No.4, July 2005.

[7] Don J. Torrieri, "Statistical Theory of Passive Location Systems", IEEE Trans. on Aerosp. Electron. Systems, Vol. AES-20, No. 2, pp. 183-198, March 1984.

[8] Chan F.K.W., So H.C., Zheng J. and Lui K.W.K., "Best Linea Unbiased Estimator Approach for Time-Of-Arrival Based Localisation”, The Institution of Engineering and Technology Signal Processing, Vol 2, 2, 2008.

[9] René Játiva E., Josep Vidal, "Estimación del Tiempo de Llegada en un canal Rayleigh desde una perspectiva de la Cota Inferior de CramerRao”, Revista Avances en Ciencias e Ingenierías, pp. 5-10, Abril 2009.

[10] René Játiva, David Sánchez and Josep Vidal, "NLOS Mitigation Based On TOA for Mobile Subscriber Positioning Systems by Weighting Measures and Geometrical Restrictions”, IEEE Asia-Pacific Conference on Computer Aided Systems Engineering, July, 2015.

[11] Menni T., Chaumette E., Larzabal P., "Reparameterization and constraints for CRB: duality and a major inequality for system analysis and design in the asymptotic region”, IEEE International Conference on Acoustics, Speech and Signal Processing (ICASSP), 2012. DOI: 10.1109/ICASSP.2012.6288682.

[12] Xiao Li, Yik-Chung Wu and Erchin Serpedin, "On Performance Bounds for Timing Estimation under Fading Channels”, IEEE Wireless Communications and Networking Conference (WCNC), 2009. DOI: 10.1109/WCNC.2009.491784. 